\title{
COMPUTERS IN INDUSTRY
}

\section{The application of STEP in the automotive supply chain}

\author{
Erick Haag $^{\mathrm{a}, *}$, Regine W. Vroom ${ }^{\mathrm{b}}$ \\ "TNO Institute of Industrial Technology, P.O. Box 5073, 2600 GB Delft, The Netherlands \\ ${ }^{\mathrm{h}}$ Faculty of Inaustrial Design Engineering, Delft Unicersity of Technology, Jaffalaan 9, 2628 BX Delft. The Netherlands
}

Received 16 August 1995; revised 10 January 1996; accepted 22 March 1996

\begin{abstract}
To improve the possibilities for the electronic exchange of product information, European car makers are involved in the development of STEP (Standard for the Exchange of Product Model Data). It is likely that STEP will be implemented within the automotive industry soon. TNO Product Centre and the Faculty of Industrial Design Engineering of Delft University of Technology, The Netherlands, jointly initiated a research project to find out if STEP is also applicable within the automotive supply chain. Data were gathered from automotive suppliers by the use of interviews and a questionnaire. The survey showed, that most of the problems with product data exchange between suppliers and customers relate to different organizational patterns for product development. Four types of suppliers are presented, each having specific requirements according to product development organization and product data exchange. STEP, which applies to the technical problems with product data exchange, can only be used effectively when the organizational problems with product development processes have been triggered.
\end{abstract}

Keywords: STEP; Application protocol; Automotive industry; Suppliers; Co-makership; Main suppliers; Product development

\section{Product data exchange in the automotive indus- try: Global framework}

\subsection{Introduction}

In recent years, development and manufacturing processes in the automotive industry have been radically changed as a result of new competitive challenges. Yet, the 1990s seem to bring no halt to the trend. This article handles the consequences of changes in product development for product data

\footnotetext{
${ }^{*}$ Corresponding author. Email: haag (àprc.tno.n!
}

exchange between car manufacturers and their suppliers. More specifically, we will look at the application of information technology to support this product data exchange.

First, we will briefly state the trends in the international car market and the business requirements imposed upon the industry. Resulting changes in the automotive supply chain will be explained. The consequences of these changes for product data exchange between supplicrs and car manufacturers will be stated. The data exchange standard STEP will be presented as a new and more structural approach to product data exchange. The second part of the article describes the results of a field survey. The survey has been executed in the period of February 1994 to 
January 1995 by TNO Product Centre in cooperation with Delft University of Technology, The Netherlands. It aimed at the assessment of problems with product data exchange in the automotive industry in The Netherlands, and the applicability of STEP to solve them. In the third part of the article, the conclusions of the field survey will be presented.

\subsection{Trends in the international automotive industry}

Four important trends have influenced the international automotive industry during the late 1980 s and early 1990s. They inflicted severe pressure upon car manufacturers and suppliers.

1. Regionally oriented competition between car makers in the USA, Europe and Japan evolved to global competition between large automotive companies from all over the world. This has resulted in a broader orientation of car manufacturers regarding the development of markets and the establishment of new production sites.

2. Sophisticated consumer demands are forcing car makers to reduce time to market and to improve total product quality.

3. The decrease of market growth and the changing view on environmental effects of motor vehicles result in new drives for technology development. Where the application of new technologies used to be an important selling force (technology push), technology development nowadays is more and more directed by consumer demands (market pull).

4. Customer-supplier cooperation has increased as a result of the growing need for production flexibility.

\subsection{Consequences of trends for the supply chain}

The trends mentioned in the preceding section have caused structural changes in the automotive supply chain. Not only do car makers try to outsource more activities to suppliers (early supplier involvement); they also reduce the number of suppliers to facilitate purchasing management. This has resulted in a more structured supply chain with suppliers in the first tier being responsible for the development and supply of complete sub-assemblies.
Smaller suppliers move to the second and third tier to carry out technological specialized activities for first tier suppliers.

\subsection{Communication within the supply chain}

To practise intensive and long-distance collaboration in product development, effective communication of product data is crucial. Drawings, specifications, files, measuring reports, and other documents necessary to create the product have to be in the right place at the right time and in the right format. With information technology tools used in virtually all disciplines of the development process, a lot of information can be exchanged directly between computer systems. However, the electronic exchange of product data turns out to be quite hazardous as every computer system uses its own specific data definition structure. To avoid the need for the development of unique translators for every pair of applications, several neutral exchange formats have been developed. But even with these formats, of which IGES, DXF and VDAFS are widely used within the automotive industry, accurate data exchange is still troublesome. This is due to ambiguous definition of the exchange formats, support of different sub-sets of product data, lack of possibilities to incorporate user specific information (e.g. user defined line-types or layers) and insufficient quality of pre- and postprocessors.

\subsection{A new approach to product data exchange}

In 1984, the International Organization for Standardization (ISO) started the development of International Standard 10303, which was named STEP (Standard for the Exchange of Product Model Data). STEP will provide a standardized method to define all data necessary for the description of a product throughout its life cycle. This creates the possibility to exchange product data among different computer systems. Several national standardization organizations and industrial circles from all over the world are involved in the development of STEP.

The need for STEP stems from the shortcomings of the neutral exchange formats as described in the preceding section. To overcome them, STEP is based 
on a more fundamental approach to product data exchange. Within STEP three main groups of standards are developed.

1. The Integrated Resources are the fundamentals of STEP. They consist of conceptual models, built according to a modular structure. These models describe technical data elements with their relationships and can be used for the description of product data in the Application Protocols.

2. Application Protocols are the fundamentals of the practical implementation of STEP. They describe a combination of data elements from the Integrated Resources for a particular industrial field or application area. Application Protocols for industrial fields have been called "vertical" APs because they support dala exchange within the whole development and engineering process of a specific sector of industry. An example of this type of Application Protocol is AP 214 for the automotive industry, which will be explained later on. Application Protocols which support a specific application area have been called "horizontal" APs. These Application Protocols provide a framework for data exchange in a restricted phase of the development process across several fields of industry. An example is AP 201, which supports data exchange for two-dimensional drawing. To describe the clata structures within the Application Protocols in a computer interpretable way, the data definition language EXPRESS is developed.

3. Implementation Forms are the physical forms for implementation of STEP in information technology systems. The most important ones are the STEP physical file format and the Standard Data Access Interface (SDAI). The STEP physical file format is a standard ASCII file format based on the data description in EXPRESS. The structure of the EXPRESS dala definition language also allows Application Interpreted models to be converted into a format for a shared database. SDAI is an EXPRESS oriented interface protocol, which allows to manipulate data independent from their database-specific format.

STEP thus provides a mechanism for the description of product data in development or production processes. Even though it takes a long time to develop a certified international Application Protocol as a part of the ISO standard STEP, the method can also be used to develop standards on inter- or intracompany level.

\subsection{STEP in the automotive industry: Application Protocol 214}

Because of the dependence on information technology systems and the intensive exchange of product data in the automotive industry, car manufacturers are actively involved in the development of STEP. Besides initiatives in this field in the United States and Japan, several European companies cooperate to develop STEP applications. In 1991, five German organizations in the automotive and electrotechnical industry started ProSTEP: "Development of Methods and Tools for Computer Aided Design and Production Facilities using STEP". ProSTEP is an organization in which more than a hundred European automotive companies jointly develop STEP standards aiming at the introduction of these as soon as possible. The most important activity is the development of Application Protocol 214: Core Data for Automotive Mechanical Design Processes. This Application Protocol provides a structural data model in which all product data, necessary for definition, styling, design, evaluation, production planning, tool design, tool manufacturing and quality control, can be stored. AP 214 provides a common framework for the storage and exchange of product data to support the automotive development process. It is planned to be released as an international standard in 1996.

\section{Survey on product data exchange in the Dutch automotive industry}

\subsection{Problem description and project goal}

As a knowledge centre of product data technologies and STEP, TNO Product Centre is interested in the application of STEP in industrial practice. Most European car manufacturers support the development of STEP and the automotive industry is likely to implement Application Protocol 214 soon. However, automotive suppliers have not yet been actively involved in the STEP activities. As their contribution 
to the development of new products is of growing importance, it seems relevant to ask if STEP, and AP 214 in particular, is also applicable for suppliers, with probably different organizational patterns of product development. TNO Product Centre and the Faculty of Industrial Design Engineering of Delft University of Technology jointly initiated a research project to find answers to the following questions.

1. Which groups of first ticr supplicrs can be distinguished in The Netherlands?

2. Which differences exist in organization of product development between these supplier groups?

3 . Which problems do the supplier groups experience with conventional (drawings, text documents, etc.) and electronic (CAD models, text files, etc.) product data exchange within product development?

4. How do problems with product data exchange relate to differences in organization of product development processes?

5. To what extent and how can STEP contribute to the solution of these problems?

\subsection{Gathering data}

To answer the research questions formulated above, data have been gathered from automotive suppliers in The Netherlands. Based on several sources, an overview was made of companies involved in automotive activities. This resulted in a list of 50 to 60 companies. Data were collected from the supplicr population by interviews and a questionnaire.

The interviews focused on product development organization of five selected suppliers. They all operate in the first tier of the supply chain. Issues of interest were: identification of start and end of the product development process, sequence and contents of activities, and departments involved. Furthermore, the information exchange between suppliers and cus-

\footnotetext{
${ }^{1}$ Product development was defined as the whole of activities necessary to start the production of a new or severely altered product. This includes all activities until the first production batch (job 0): concept development, product design, product engineering, tool development, tool production, prototype building, prototype testing, production planning.
}

tomers was charted: frequency and type of contact with customers, and input and output information of each step in the development process.

The data collected in the interviews were verified by a questionnaire among the supplier population. Besides general business information, companies were questioned about the type of contact with customers in product development. An assessment was made of the stages in product development and of the contents and intensity of product data exchange. Suppliers were also asked about the problems they experience with the exchange of product data. The response to the questionnaire covered about $20 \%$ of the automotive supplier population in The Netherlands.

\subsection{The field survey}

The most remarkable result of the interviews is, that the way in which suppliers collaborate with customers in product development projects varies extremely. Two characteristics seem to be of influence here.

First, the technical complexity of the supplier's product determines the contents of information to be exchanged and, consequently, the accuracy of cooperation needed. A tow bar manufacturer, subjected to the interviews, produces a technically not very complex product. He only needs information about fastening points on the car and room for the tow bar construction which can be derived from product drawings. A dashboard supplier, on the other hand, makes a very complex product with a sophisticated interface to the vehicle. To match all components, a lot of information is needed. Therefore, this company's initial product information consists of a complex three dimensional CAD model in which the dashboard assembly is inserted. This characteristic can be expressed by the distinction of main suppliers and co-suppliers, with main suppliers delivering complex and independent units or sub-assemblies and co-suppliers delivering parts or components which have to be assembled with other less complex products of co-suppliers.

The questionnaire provided characteristic differences between main suppliers and co-suppliers. Fig. 1 depicts, for twelve of the suppliers questioned, the amount of customers in the automotive industry, the 
percentage of quotations accepted and the percentage of the turnover obtained from activities for automotive customers. The activities of the companies numbered 10,11 and 12 , all being main suppliers, focus on the automotive sector. Furthermore, the main suppliers have less customers than co-suppliers numbered 5 to 9 (the amount of automotive customers for remaining co-suppliers 1 to 4 is relatively low because they also work for other sectors of industry). Finally, the figure shows that relatively few quotations of main suppliers are accepted by customers. This can be explained by the severe competition among main suppliers according to the acquisition of new product development contracts.

The second characteristic influencing the cooperation between supplier and customer is the amount of development phases in which the supplier is involved. Where, for example, the interviews showed a supplier of sheet metal body parts only carrying out advanced engineering and production activities, a dashboard supplier is actively involved in the whole development process of his customer. Initial product information for the sheet metal specialist consists of released drawings or CAD models, which can hardly be influenced. The initial information for the dashboard supplier is a list of functional requirements

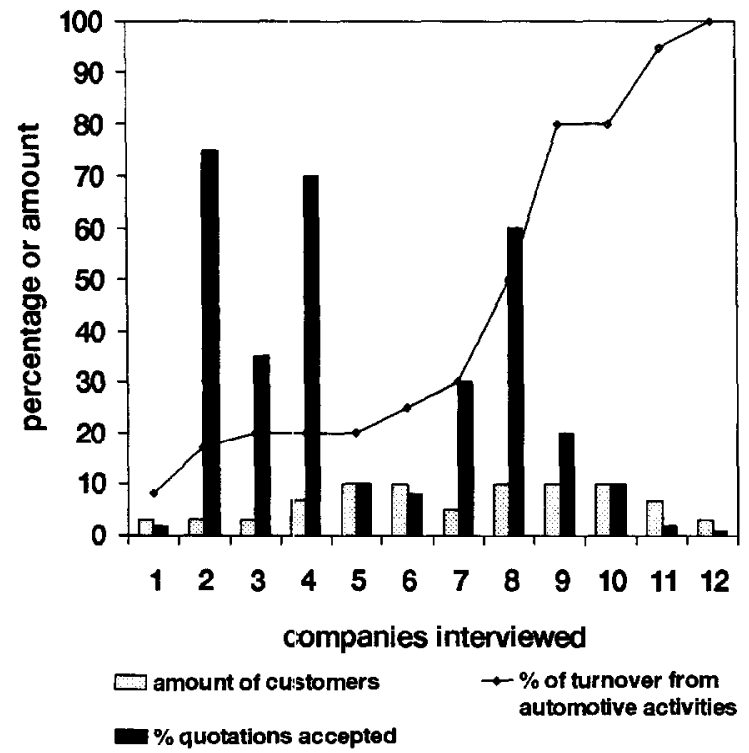

Fig. 1. Amount of customers, quotations accepted and activities for the automotive industry of twelve companies questioned.

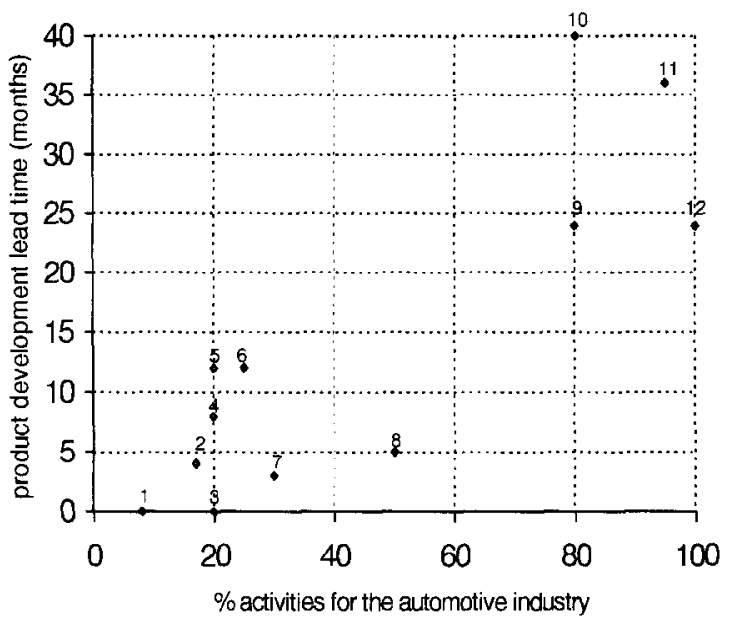

Fig. 2. Lead time of product development process related to the percentage of activities for the automotive industry.

and initial CAD models of the vehicle, which he uses to develop the dashboard in close cooperation with the customer. In general, two types of cooperation are distinguished according to the product development stages in which the supplier takes part. A co-developer is involved in almost all stages of product development, as he designs and engineers the product, and additionally carries out engineering and production activities. A co-maker, on the other hand, carries out product engineering activities and only indirectly influences the product design by advising the customer about production requirements. A third supplier type distinguished according to this characteristic is the jobber, who is not involved in product development at all, but only carries out production and process engineering activities. This supplier type is of less interest here, as product data exchange between jobbers and car manufacturers will be very limited compared to the other supplier types.

In Fig. 2, an overview is given of the product development lead time of companies subjected to the questionnaire. The graph shows that companies working mainly for the automotive industry (suppliers 9, 10, 11 and 12), have longer development processes than other suppliers. These suppliers actually cooperate with their customers in product development projects. Except for company 12, which is a production plant of a co-developing supplier in the 
UK, these companies might be called co-developers. With suppliers 1 and 3 being jobbers, we see that most of the suppliers in The Netherlands can be labelled co-makers, advising their customers in the product development and engineering process.

The two characteristics mentioned in this section can be combined to form a general model for supplier classification, which will be explained later on.

\subsection{Supplier classification and product development organization}

Basically, for the researched companies, the product complexity relates to the intensity of contact with the customer in a single project. Main suppliers have a more frequent contact with their customers than co-suppliers. When the development process is graphically modelled in a flow-chart (of activities, information flows, and "go"/ "no-go" decisions), we see that co-suppliers have much longer "loops" in their processes than main suppliers (see Fig. 3): with frequent feed back information from customers, only few activities have to be repeated in case of a "no-go" decision. As a result, the process organization of the main suppliers is adjusted very well to the development process of customers. The dashboard supplier, mentioned earlier, organizes his development process according to very specific requirements of the customers. On the contrary, co-suppliers organize their development processes according to the requirements of their, often technologically specialized, production process. The tow bar supplier, for example, adjusts his development process to the very specialized activities of designing and testing tow bar assemblies. This is also reflected in the management of product development projects. The dashboard manufacturer and a supplier of exhaust systems manage product development through a product manager, who has extensive power to influence the project. For the suppliers of tow bars and sheet metal components, the product manager's responsibility is mainly to coordinate the activities of different departments.

The second dimension for the supplier classification, the stages of product development in which the supplier gets involved, relates to the length of cooperation between customer and supplier. As car manufacturers try to outsource more and more of their non-core activities, co-developers gain an important position within the supply chain. Not only do car makers cooperate with them in various stages of the project, often a partnership is being developed over a longer period of time. Of course, mutual thrust is a condition to make such partnerships work, and detailed agreements should be made on milestones and deliverables of development phases, procedures for
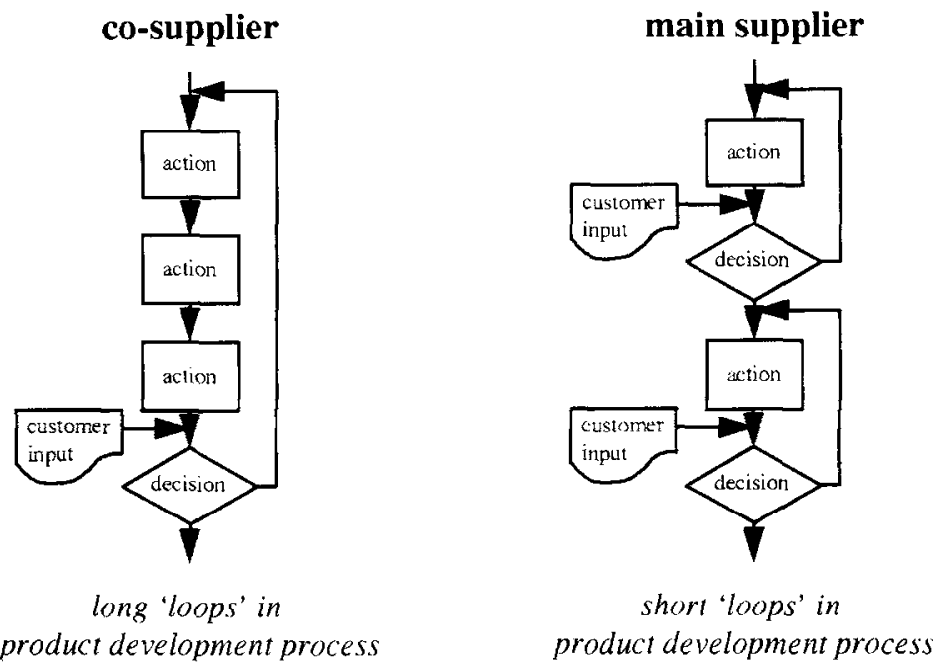

Fig. 3. Schematic product development charts of co-supplier and main supplier. 
communication, applicability of supplier's technologies for other customers, division of revenues resulting from learning curve advantages, etc.

Fig. 4 shows the amount of face-to-face contacts between automotive suppliers subjected to the questionnaire and their customers in several stages of product development. First, this graph shows that co-developing main suppliers (numbers 10 and 11) maintain a more frequent contact with customers than the other companies. Second, the figure also illustrates that co-developers (numbers 9, 10 and 11) maintain contact with their customers during all stages of the development process, whereas comakers mainly see their customers during the development and engineering stage. Main supplier 12 does not maintain frequent contact with customers because the product development work is done by a separate division in the UK.

\subsection{Product data exchange in practice}

From the results of the questionnaire, an overview is derived of product information being exchanged with customers in product development projects (Fig.

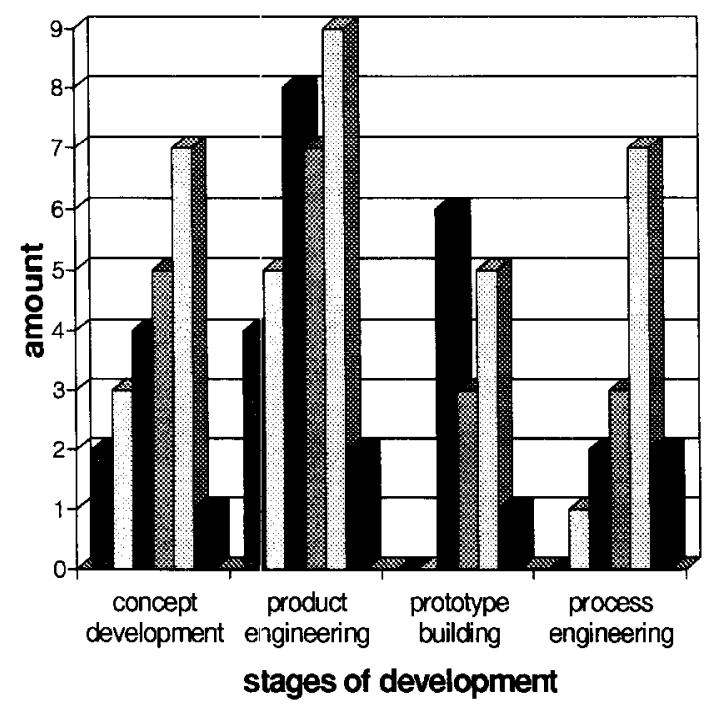

\section{D company 5 \& company 10 口company 7 Elcompany 11 - company 9 I company 12}

Fig. 4. Face-to-face with costumers in main stages of product development.

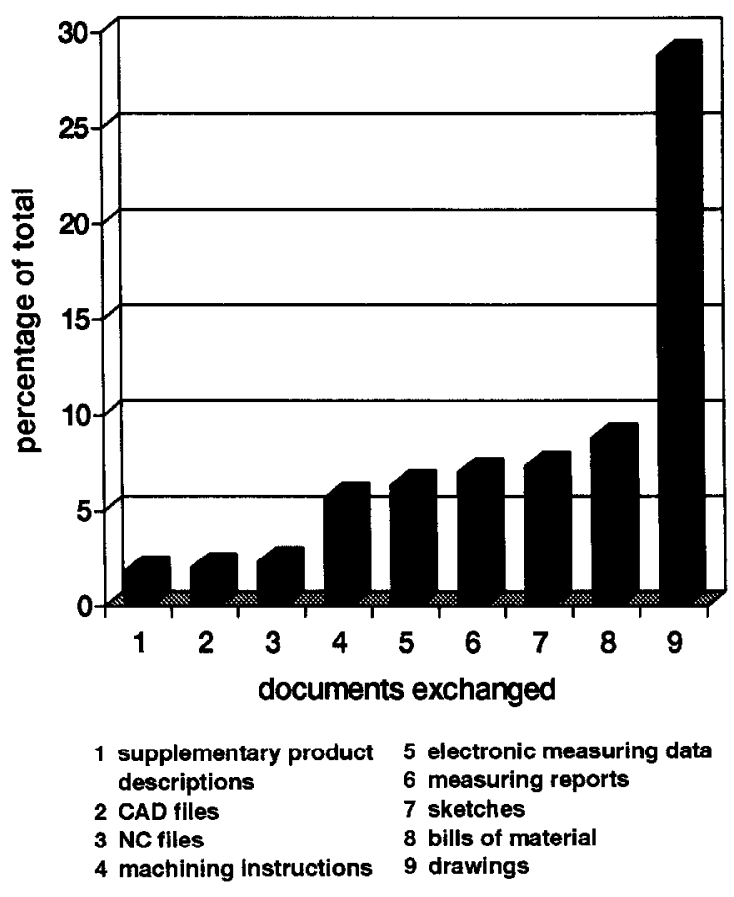

Fig. 5. Product documents exchanged by automotive suppliers.

5). It shows that product information is mainly communicated by drawings and written documents. Electronic data exchange is not yet very common in the Dutch automotive supplier industry. A lot of companies have the technical means for electronic data exchange (CAD systems, pre- and post-processors for neutral data exchange file formats), but do not use them effectively. But also by using conventional data exchange techniques (e.g. drawings), communication between customer and supplier is not always smooth.

The rating of suppliers in the two dimensions of supplier classification can be used to chart the data exchange problems (see Fig. 6).

Referring to the dimension of product complexity, we see that suppliers of relatively simple products (co-suppliers) experience many problems with data exchange. A typical problem is initially released product information being changed by the customer later on; co-suppliers often experience change requests relatively late in the development process. Another problem is product data being insufficient to be used effectively by the co-supplier. Geometry information, for example, might be incomplete or 
unsuitable for the supplier's purpose (tolerances, surfaces, etc.). Most of these problems are caused by differences in organization of the development process between co-supplier and customer. The co-supplier uses his technological know-how to do specialized work for a lot of customers in several sectors of industry. The way in which product development and production are organized has evolved over the years according to requirements of the production technology. Planning, milestones, activities and information flows might not fit the characteristics of the customer's process. Usually, co-supplier and customer are unaware of each others activities and progress. Product data will turn out to be incomplete, unsuitable, too late, or in the wrong format.

Main suppliers in the automotive industry generally experience other types of problems when they exchange product data with customers. Typical examples are differences in interpretation of data and version management. For main suppliers who transfer data electronically, problems result from obscurity about the legal status of data, loss of data with file conversion and the limited applicability of neutral file formats. Most of these problems are not due to organizational mismatches, but to technical difficulties in the tools and procedures for data exchange or in the product itself. Main suppliers are aware of milestones, activities, and input and output information of their customer's process. Thus, main suppliers have to cope with problems mainly concerning the tcchnical content of product information.

As the second dimension for supplier classification relates to the length of the relationship between supplier and customer, it indicates whether investments in product data exchange will be relevant. When a substantial contribution to product development is made by the supplier (co-development), requirements for information exchange will be severe. Especially in case of long-term partnerships, it is useful to pay considerable attention to the development of detailed procedures for data exchange, e.g. according to drawing conventions, formats, and authorization. For more complex products, it might be relevant to invest in tools, e.g. CAD systems, data

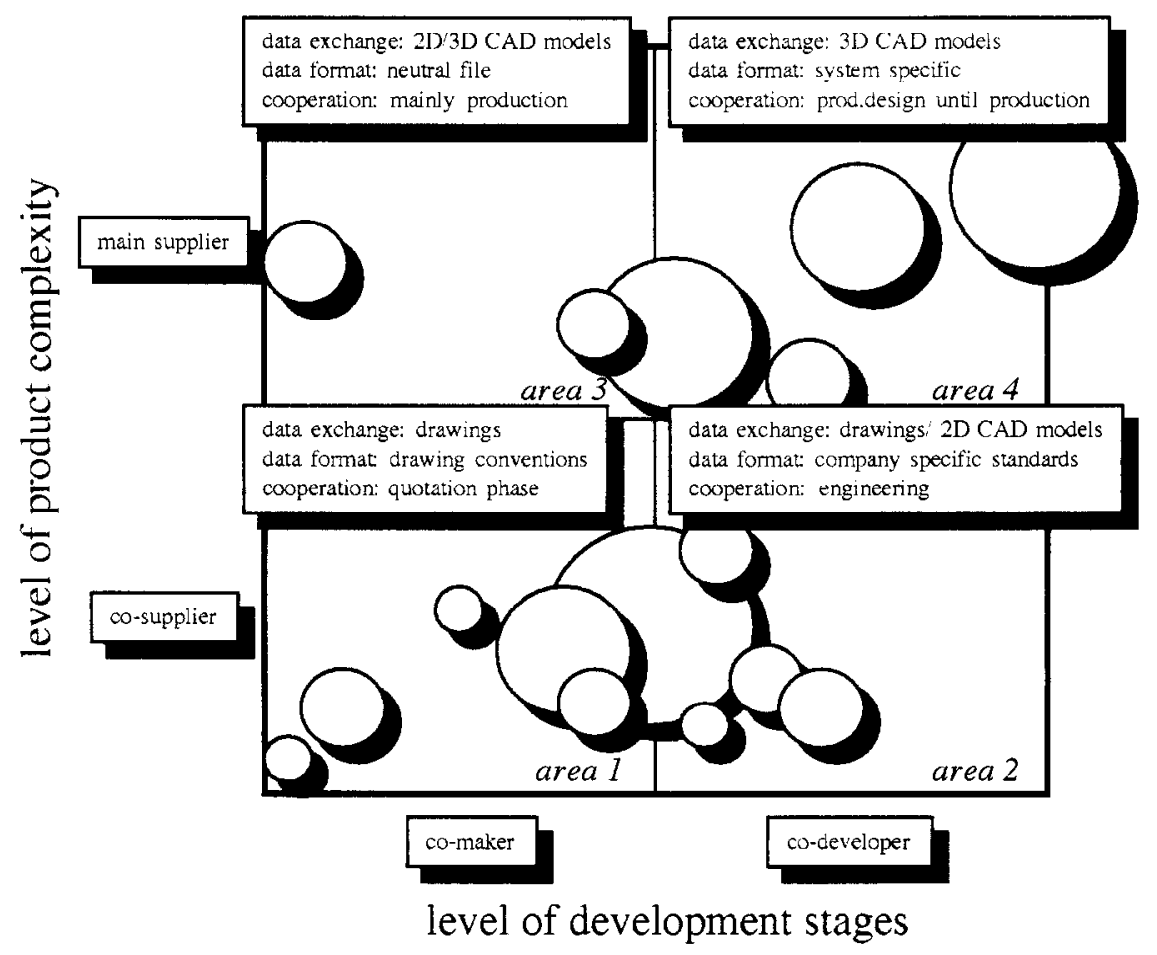

Fig. 6. Characteristics of product development and product data exchange dependent on dimensions of supplier classification. 
exchange formats and file translators. When the supplier-customer contact in a product development project is only temporarily and focuses on few process steps, agreements should focus on what information is needed and how it can be exchanged with tools and methods presently available within both companies.

Now that problems with product data exchange are generally classified, wc can conclude that the solution for a lot of problems might be primarily found in the improvement of organizational patterns of product development. Only when processes of customer and supplier match to some extent, it will be useful to concentrate on advanced technical solutions for improvement of product data exchange. Where companies often experience problems with data exchange formats (e.g. IGES, DXF), computer systems (e.g. CAD systems), file translators (e.g. pre- and post-processors), the underlying organizational procedures often have not yet been defined. A first step to improve data exchange for the supplier will thus be to recognize the position of the company on both dimensions of classification (expressed as the complexity of the product and amount of stages of product development). In the next section, this will be explained further.

\section{Results}

\subsection{Supplier classification matrix}

In Fig. 6, the classification of suppliers is expressed as a matrix. The vertical axis schematically depicts the level of product complexity of suppliers subjected to the field study, reaching from simple products like nuts and bolts to very complex products like dashboard sub-assemblies. The horizontal axis reflects the stages of the product development process in which the supplier is involved. This axis reaches from solely production to complete product development and manufacturing (concept development, design, engineering, prototyping, tooling, and production). All suppliers subjected to the study, are reflected in the matrix with the size of the circle giving an indication of the company's yearly turnover.

Using the supplier types main supplier, co-sup- plier, co-developer, and co-maker, the matrix can be divided into four areas, with general characteristics according to product development and product data exchange with customers in the automotive industry. Although boundaries between the areas will not be particularly clear in reality, they are drawn in the figure to clarify the idea. The characteristics of every area will be mentioned below.

First area: Low product complexity and few stages in product development.

- Short-term contracts for a fixed batch size or a fixed time period.

- One or few customers in the automotive industry; activities also focus on other industry sectors.

- Mainly two-dimensional product data being exchanged by the use of paper drawings.

- Hardly any agreements regarding planning and procedures for product development and data exchange; the customer dictates drawing conventions and provides detailed specifications for price, quality, delivery time, etc.

- Contact between supplier and customer concentrates on the initial development (quotation) phase, in which negotiations on price and delivery time take place. The supplier only plays a very limited role in the actual product development process.

Second area: Low product complexity and many stages in product development.

- Medium-term contracts for a fixed batch size or occasionally life time contracts in which delivery during the whole life cycle of the end product is guaranteed.

- Relatively large amount of automotive customers; there is generally no capacity to work for other sectors of industry because the supplier's process is tuned to the automotive industry.

- Product data exchanged with customers are mainly two-dimensional; drawings as well as two-dimensional CAD models are being used.

- Agreements between customer and supplier relate to drawing conventions or neutral data exchange formats to be used for electronic data exchange; usually, these conventions are prescribed by the customer.

- Contact between supplier and customer concentrates on the engineering phase, in which technical specifications of the supplied product are being determined. The supplier usually gets in- 
volved immediately after the functional specifications of the supplier's product are captured.

Third area: High product complexity and few stages in product development.

- Long-term contracts for a fixed batch size or life time contracts.

- Small amount of customers in the automotive industry; the turnover of the suppliers in this group is generated mainly by activities for this sector.

- Product data are complex and therefore three-dimensional CAD models or prototypes are being exchanged in addition to detailed technical drawings.

- Agreements on procedures for product data exchange usually are being laid down in the contract. In many cases, the customer sets requirements for the file format to be used. Because the supplier usually works with a different CAD system than the customer, neutral data exchange formats like IGES or VDAFS will be used. The supplier is responsible for conversion of electronic data.

- Contact with the customer concentrates on the production phase: the exchange of production planning data, often used for the purpose of justin-time delivery, is much more intensive than product data exchange in product development.

- Most of the suppliers in this category are engineering or production divisions of larger organizations, which have their product development activities concentrated in a scparate division.

Fourth area: High product complexity and many stages in product development.

- Usually, life time contracts are appointed because the supplier has to make considerable costs to develop the initial product concept; new projects are acquired by concept competition: several main suppliers compete for a contract by submitting an initial product proposal to the customer.

- Very limited amount of customers (one to five); severe requirements of customers force main suppliers to adapt their business processes completely.

- Detailed agreements according to planning and procedures for product development and product data exchange; issues of interest might be: quality aspects, milestones, CAD/CAM systems and in- formation carriers to be used, technologies incorporated, etc. In many cases, the customer demands from the supplier to use a specific CAD system to guarantee successful exchange of CAD models.

- The suppliers within this category are closely tied to their customers, not only in product development but also in the production phase; logistic and quality systems are usually integrated with $\mathrm{EDI}^{2}$ and total quality management techniques.

\subsection{Data exchange problems}

Looking at the matrix of Fig. 6, we see that especially co-developing main suppliers in the fourth area are faced with severe requirements of car manufacturers. These suppliers are forced to fully adjust the organization of their development processes, but also their systems and data formats, to their customers. They need to use different systems and data formats to serve several car manufacturers. Additionally, they are likely to face data exchange problems with their own suppliers, which often are co-suppliers in the second and third area of the matrix. Co-suppliers, with technological specialized development processes and systems, lack knowledge and means to exchange product data with main suppliers electronically. Thus, co-developing main suppliers face most of the difficulties related to the electronic exchange of product data. They will have to play a leading role to introduce and improve electronic data exchange in the automotive supply chain.

\subsection{Applicability of STEP}

As the results of the field survey showed, the applicability of STEP as a data exchange standard in the supply chain is restricted. STEP can only provide a solution for the technical probleniss with product data exchange, whereas most of the problems are due to differences in organization of product development. Referring to the matrix of Fig. 6, STEP - and Application Protocol 214 in particular - applies

\footnotetext{
${ }^{2}$ EDI: Electronic Data Interchange: electronic exchange of administrative data e.g. for order management or production planning.
} 
mainly to the co-making and co-developing main suppliers in the third and fourth area. These companies supply complex products and have to use structural procedures for product data exchange to successfully develop products for their customers. Using STEP instead of existing data exchange formats will provide the possibility to exchange more types of data.

Particularly companies in the second and third area of Fig. 6, who serve a relatively large amount of customers, will value STEP as a solution for data exchange problems. Their customers often use different CAD-systems and have various requirements according to data formats. However, this group of suppliers will only require limited use of STEP data models, because they need (and are allowed to have access to) a limited amount of product data.

Thus, the "vertical" Application Protocol 214, especially designed for application in the automotive industry, is likely to suit main suppliers better than co-suppliers. For specialized suppliers in the second and third area, "horizontal" Application Protocols, which are designed to support data exchange within specific development activities, seem to be more suitable. From this perspective, main suppliers will form the link between data exchange supported by "horizontal" Application Protocols and data exchange supported by the "vertical" Application Protocol 214.

\section{Conclusions}

The most important conclusion to be drawn from this survey is that the applicability of STEP in the automotive supply chain is restricted. Most of the problems with product data exchange are caused by managerial mismatches in product development and engineering. To make use of STEP effectively, customers and suppliers have to define procedures for product development, process planning, and communication. Only after that, organizational procedures and technical solutions for product data exchange can be implemented and used successfully.

As a second conclusion, we can state that co-developing main suppliers will have to play a key role in successfully introducing and improving the electronic exchange of product data in the automotive supply chain. This is due to their intermediate role between co-suppliers of tcchnologically spccialized parts and car manufacturers who assemble the final product.

The third and final conclusion deals with the development of STEP as a neutral data exchange format. We recognized different kinds of Application Protocols and divided them into "horizontal" APs, supporting data exchange in particular activities across several fields of industry, and "vertical" APs, supporting data exchange in the development and manufacturing processes of specific sectors of industry. As both types of APs are suited for different organizations within the automotive supply chain, it is important to clearly define the interfaces and overlapping areas between both types of APs within the continuing development of STEP.

\section{For further reading}

Clark and Fujimoto, 1991, Haag et al., 1994, Haag, 1994, Kuiper, 1993, Mohrmann and Speck, 1994, ProSTEP Centre, 1993, Ranke, 1989, Womack et al., 1991

\section{References}

K.B. Clark and T. Fujimoto, Product Development Performance: Strategy, Organization, and Management in the World Auto Industry, Harvard Business School Press, Boston, 1991.

E.J. Haag, R.W. Vroom and A.E. Vries-Baayens, "Problems in Exchanging Product Information between Automotive Suppliers and Car Manufacturers", Proceedings of the 27th International Symposium on Automotive Technology and Operation; Dedicated Conference on Lean and Agile Manufacturing, Aachen, 1994.

E.J. Haag, "Uitwisseling van produktinformatie in de Nederlandse automobielbranche", TNO Report 94-CAD-R0033, TNO Product Centre, Delft, The Netherlands, 1994.

P. Kuiper, STEP: Een inleiding, Instruction for Workshop Software and SIEP, INO Centre for PDI and CALS, Delft, The Netherlands, 1993.

J.M. Mohrmann and H. Speck, Application protocol: Core Data for Automotive Mechanical Design Processes, Working Project Draft of ISO 10303, Part 214. ISO TC184/SC4/WG3 N331, Daimler-Benz/ CAP Debis, Leinfelden, 1994.

ProSTEP Centre, ProSTEP; System Integration with STEP, Berlin, 1993.

A.A.M. Ranke, Introduction to STEP, Series of Standards for 
Product Data Representation and Exchange, Philips Research Laboratories, Sector Information and Software Technology, Eindhoven, The Netherlands, 1989.

J.P. Womack, D.T. Jones and D. Roos, The Machine that Changed the World, the Story of Lean Production, Harper Perennial, New York, 1991.

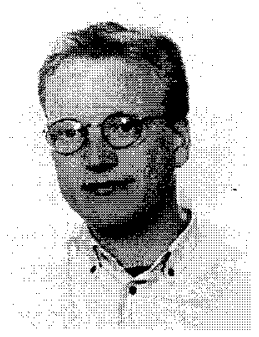

Erick Haag graduated at the Faculty of Industrial Design Engineering of Delft University of Technology. At present, he is working as a Project Engineer at the Division Product Development of TNO Institute of Industrial Technology. $\mathrm{He}$ is involved in research and consultancy projects to improve customer-supplier relations within product development processes. $\mathrm{He}$ also conducts courses for industrial companies to provide information on the applicability of the data exchange standard STEP.

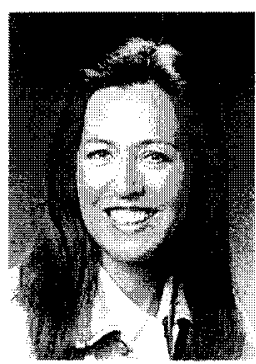

Regine W. Vroom graduated at Delft University of Technology, Faculty of Industrial Design Engineering. She has worked as a CAD analyst at Volvo Car in The Netherlands, now part of the company NedCar. Since 1987, she is Assistant Professor at Delft University of Technology. She lectures design engineering and a course called "Technical Product Information". Her research activities include a doctoral research on the passing and feedback of information within engineering and between engineering and other disciplines of Dutch automotive suppliers. Further, Regine Vroom is a member of the Board of the Faculty, she is active as a reviewer for the Esprit Programme of the European Community and she is a member of the Dutch Product Data Management (PDM) platform. 